will the program impact lifestyle and lay the foundation for good health, a decent quality of life and wellbeing.

From the vision, the mission will be derived as to the targets; how they will they be measured and over what time period. The values of the program has to be shared where workers and the employer agree and work together to achieve common goals.

To strategically attain work life balance; a principled health and wellness programs has to be developed and integrated into the business operations as a key performance indicator. By so doing the programs will be aligned to the business strategies.

Results With this alignment some of the possible results that could be realised are:

- A culture of safety

- Reduction in on the job accidents

- Increased productivity and reduced absenteeism

- Reduction in sick days leading to reduction in utilisation of health insurance scheme

- Improved profit margins

- Improved brand image - employer of choice

- A more satisfied employee

- A changed worker with improved attitude and behaviour

Discussion Many blue chip companies in developed countries have established programs in place to achieve work-life balance, health and wellbeing for their staff. Some of these companies are Google, Alcoa, Fiat and Virgin Airlines, to name a few well know ones. Paul O'Neill, former CEO of Alcoa in a CNN May 2014 interview spoke about safety as a priority at Alcoa. It could be gleaned from his remarks that work life balance was achieved from the involvement of management and their commitment to a vision. Also that the desired values and attitudes for a culture of safety, work-life balance, health and wellbeing were cascaded down to the workers from the strategic level and were accepted by the employees.

In summary, Work-Life Balance Health and Well-Being is not an insurmountable goal. To support the achievement of this balance for workers, the ILO under its pillar of Decent Work has a health and safety convention that serves as an international standard for nations to pattern in their national programs. In this respect, legislations have been passed in many countries in support of the Convention. It is of note that European countries in support of the promotion of workers health and wellbeing have passed legislations. The European Framework Agreement on work-related stress was one such legislation that was passed on 8 October 2004 .

There is also the Italian Regulatory Framework for Health and Safety in the Workplace, Legislative Decree 81/08. This was bench marked form the UK Health and Safety Management Standard for Work related Stress, Belgian Screening, Observation, Analysis, Expertise (SOBANE) Strategy of Risk Management and German stress-psychology-health (start).

The principle of work of work-life balance is generally accepted globally. However there is a perception of institutional inertia in many developing countries to enact the necessary legislations to support a national policy and program framework for occupational health and safety. It is hoped that developed countries that are signatories to the ILO Occupational Health and Safety Convention as well as countries in the European Unions, will extend more significant and substantial support in the areas of human resource and capacity building, as well as financial assistance to help developing countries strike that desired balance.
225

A ROLE FOCUSED SELF-HELP COURSE REDUCES STRESS AND SUBSEQUENT WORK FUNCTIONING PROBLEMS IN WORKERS WHO HAVE INFORMAL CARE RESPONSIBILITIES

EJ Boezeman, K Nieuwenhuijsen, JK Sluiter. Academic Medical Centre, University of Amsterdam, Department: Coronel Institute of Occupational Health, Amsterdam Public Health research institute, Amsterdam, The Netherlands

\subsection{6/oemed-2018-ICOHabstracts. 1628}

Introduction Many paid workers who fulfil informal care responsibilities in their private situation report stress complaints and impaired functioning at work. It was examined whether a newly developed role-focused self-help intervention decreases caregiver stress and distress, and work functioning problems, in these workers.

Methods A study that had a randomised controlled design (intervention vs wait-list control) was conducted. Workers suffering stress due to involvement in informal care activities were participants $(n=128)$. The workers allocated to the intervention group received the role-focused self-help course. The workers allocated to the control group received the intervention after all measurements. Caregiver stress (primary outcome), distress, work functioning, care-to-work interference, and care-to-social and personal life interference, were measured prior to the random allocation (pre-test) and one (posttest 1) and two (post-test 2) months after allocation. Mixed Model ANOVAs and mediation analyses were used in dataanalysis.

Results The mixed model ANOVAs showed that two months after allocation the workers allocated to the intervention group had decreased levels of caregiver stress and distress as compared to the workers allocated to the no treatment control group. Further, the mediation analysis suggested that involvement in the intervention decreases functioning problems in workers who have informal care responsibilities because it reduces caregiver stress.

Discussion The role-focused self-help course intervention decreases levels of caregiver stress and distress in workers who suffer stress due to involvement in informal care activities. Because of this effect, the intervention also plays a role in the work functioning of these types of workers. The intervention represents a contribution to the literature and a means for occupational health professionals and organisations to contribute to the health and functioning of workers who combine paid work with informal care activities

\section{HIGH BURNOUT LEVEL AS MAIN DETERMINANT OF LOW WORK ABILITY AMONG FLIGHT ATTENDANTS}

${ }^{1,2}{ }^{2} \mathrm{M}$ Milošević* ${ }^{1}, 1 \mathrm{~J}$ Mustajbegović, ${ }^{2} \mathrm{~T}$ Madžar, ${ }^{3} \mathrm{M}$ Bubas, ${ }^{4} \mathrm{~T}$ Rogina, ${ }^{5} \mathrm{~T}$ Furlan. ${ }^{1}$ University of Zagreb, School of Medicine, WHO Collaborative Centre for Occupational Health, Croatia; 'University of Zagreb, School of Medicine, Centre for Sports Medicine and Health Promotion at Work, Croatia; ${ }^{3}$ Croatian Institute for Health Protection and Safety at Work, Zagreb, Croatia; ${ }^{4}$ Varaždin County Heath Care Centre, Varaždin, Croatia; ${ }^{5}$ Adriamedic Clinic for Occupational Medicine, Pazin, Croatia

\subsection{6/oemed-2018-ICOHabstracts. 1629}

Introduction The roles of flight attendants are to provide excellent customer service to passengers while ensuring their comfort and safety throughout the flight. Flight attendants have more responsibilities than most front-line employees in the service industry, as they are first trained to maintain cabin 